\title{
Differentially methylated regions in patients with rheumatic heart disease and secondary pulmonary arterial hypertension
}

\author{
DAWEI ZHENG ${ }^{1 *}$, XIAOYING CHEN ${ }^{2 *}$, NI LI $^{1}$, LEBO SUN $^{1}$, QINGYUN ZHOU ${ }^{1}$, \\ HUOSHUN SHI ${ }^{1}$, GUODONG XU ${ }^{1}$, JING LIU $^{2}$, LIMIN XU ${ }^{2}$, SHIWEI DUAN ${ }^{2}$ and GUOFENG SHAO ${ }^{1}$ \\ ${ }^{1}$ Department of Cardiothoracic Surgery, Ningbo Medical Center, Lihuili Hospital, Ningbo University, Ningbo, \\ Zhejiang 315041; ${ }^{2}$ Medical Genetics Center, School of Medicine, Ningbo University, Ningbo, Zhejiang 315211, P.R. China
}

Received July 27, 2016; Accepted April 10, 2017

DOI: $10.3892 / \mathrm{etm} .2017 .4652$

\begin{abstract}
The aim of the present study was to identify differentially methylated regions (DMRs) in patients with rheumatic heart disease and secondary pulmonary arterial hypertension (RHD-PAH). A genome-wide DNA methylation assay was performed between 6 patients with RHD-PAH and 6 healthy controls using an Illumina Infinium HumanMethylation450 BeadChip kit. The Limma software package was subsequently used to identify significant DMRs. A total of 40 hypomethylated and 64 hypermethylated $\mathrm{CpG}$ sites were identified between the RHD-PAH group and the control group. Gene Ontology and Kyoto Encyclopedia of Genes and Genomes term and signaling pathway enrichment analyses revealed that the DMRs, mapped to the genes including protein kinase $\mathrm{C}$ $\alpha$, protein kinase AMP-activated non-catalytic subunit $\gamma 2$, sprouty related EVH1 domain containing 2 and LIF interleukin 6 family cytokine, were significantly enriched in the negative regulation of protein kinase/transferase activity and the positive regulation of protein amino acid phosphorylation/phosphate metabolic process. The identified DMRs may provide novel insights into the pathogenesis of RHD-PAH.
\end{abstract}

Correspondence to: Professor Guofeng Shao, Department of Cardiothoracic Surgery, Ningbo Medical Center, Lihuili Hospital, Ningbo University, 57 Xingning Road, Ningbo, Zhejiang 315041, P.R. China

E-mail:sgf1958@sina.com

Professor Shiwei Duan, Medical Genetics Center, School of Medicine, Ningbo University, 818 Fenghua Road, Ningbo, Zhejiang 315211, P.R. China

E-mail: duanshiwei@nbu.edu.cn

*Contributed equally

Key words: DNA methylation, rheumatic heart disease, pulmonary arterial hypertension, genome-wide

\section{Introduction}

Rheumatic heart disease (RHD) is an autoimmune inflammatory disease with multiorgan involvement (1). RHD is caused by an abnormal immune response to group A streptococcal infection (2), which leads to valve damage, particularly to the mitral valve, and hemodynamic changes (3). In China, RHD remains a significant health burden, although the prevalence of RHD has been declining in recent years (4). Valve surgery is considered the primary clinical treatment for RHD (5). However, patients with RHD frequently suffer from serious complications and irreversible valve dysfunction due to a lack of early detection (3).

Pulmonary arterial hypertension (PAH) is the most frequent clinical complication of RHD (6). Right ventricular hypertrophy and heart failure during the later stages of PAH are attributable to significant reductions in the cross sectional area of pulmonary vasculature, which may eventually be fatal $(7,8)$. PAH is a complex disease; multiple factors contribute to its onset and development, including pathological environmental factors, genetic polymorphisms and epigenetic changes (9-11). Recent studies have demonstrated the role of epigenetic modifications in the pathogenesis of PAH (11-13), suggesting that DNA methylation may be associated with the etiology of RHD with secondary PAH (RHD-PAH).

DNA methylation typically occurs at the 5 ' position of the cytosine ring in 5'-C-phosphate-G-3' (CpG) dinucleotides and is often associated with the regulation of gene expression (14). DNA methylation serves an important role in the development and progression of rheumatic diseases (15-17). However, studies investigating the role of epigenetics in RHD-PAH are scarce. The present study aimed to identify differentially methylated regions (DMRs) in patients with RHD-PAH. The present study identified novel DNA methylation markers and will aid in improving the current understanding of RHD-PAH.

\section{Materials and methods}

Sample collection, DNA extraction and bisulfite modification. The clinical diagnosis of RHD-PAH was performed as previously described (3). Specifically, the inclusion criteria were as follows: i) Diagnosis of mitral valve prolapse and scheduled for mitral valve replacement; ii) left ventricular ejection fraction 
volume $>50 \%$ and left ventricular end-diastolic diameter $<55$ $\mathrm{mm}$; iii) pulmonary artery systolic pressure $>40 \mathrm{mmHg}$ prior to surgery; and iv) no history of cardiomyopathy, congenital heart disease, liver disease or renal disease. The present study was approved by the Ethics Committee of Lihuili Hospital and informed consent forms were signed by all participants. The clinical and pathological characteristics of the involved individuals are described in Table I. Since acute rheumatic fever occurs more frequently in females (2), a total of 6 female patients with RHD-PAH (the study group; age, $57.00 \pm 8.39$ years) and 6 normal female donors (the control group; age, $55.00 \pm 6.39$ years) were recruited for the present study from Lihuili Hospital (Ningbo, China) between March 2014 and September 2015.

Blood samples from the two groups of patients were collected into EDTA tubes. DNA extraction and quantification procedures were subsequently performed as previously described (18). Genomic DNA bisulfite conversion (500 ng) was performed using an EZ DNA Methylation-Gold ${ }^{\mathrm{TM}}$ kit (Zymo Research, Irvine, CA, USA), according to the manufacturer's protocol.

Methylation assay. A methylation assay was performed using the Infinium HumanMethylation450 BeadChip kit (Illumina, Inc., San Diego, CA, USA) as described previously (19). In brief, bisulfite-converted DNA (200 ng) was used in the whole-genome amplification reaction, followed by an enzymatic end-point fragmentation, precipitation and resuspension in hybridization buffer (19). Subsequently, all steps were performed following the standard Infinium protocol. Sample labeling, hybridization to chips and image scanning were also carried out according to the Infinium kit's protocol (20). All samples were processed on a single chip to avoid the batch effect. A total of 485,577 methylation loci, covering 21,231 genes, were tested using this kit. Gene methylation datasets were submitted to the Gene Expression Omnibus (accession number GSE84003).

Statistical analysis. Statistical analyses were conducted using $\mathrm{R}$ software version 3.0.1 (www.r-project.org). Methylation levels of $\mathrm{CpG}$ sites are expressed as $\beta$ values ranging from $0-1$. Methylation levels were measured using $\beta$-values, which are based on the fluorescence intensity of methylated and unmethylated probes. The calculation of $\beta$ values was performed as previously described (21). Prior to further calculations, probes designed for sequences on sex chromosomes, with random single nucleotide polymorphisms in the probe-mapping genomic regions or with detection values of $\mathrm{P}>0.01$ on all arrays were removed. The remaining 463,289 probes were used for subsequent analysis. Methylation levels were then compared with the differential detection procedure in the Limma package version 3.30.13 (www.bioconductor. org/packages/release/bioc/html/limma.html) using R software, as previously described (21). $\mathrm{P}<0.05$ and an absolute value of $\Delta \beta>0.2$ were considered to indicate a statistically significant difference. The selection process is presented in Fig. 1. IlluminaHumanMethylation450k.db annotation package version 2.0.9 (http://www.bioconductor.org/packages/release/data/annotation/html/IlluminaHumanMethylation $450 \mathrm{k} . \mathrm{db} . \mathrm{html}$ ) was used to provide detailed information about
Table I. Clinical data for patients with rheumatic heart disease and secondary pulmonary arterial hypertension and healthy controls.

\begin{tabular}{lccc}
\hline Subgroup & Age (years) & Gender & $\begin{array}{c}\text { PASP before } \\
\text { surgery (mmHg) }\end{array}$ \\
\hline Case 1 & 60 & Female & 42 \\
Case 2 & 56 & Female & 117 \\
Case 3 & 58 & Female & 72 \\
Case 4 & 56 & Female & 55 \\
Case 5 & 69 & Female & 50 \\
Case 6 & 43 & Female & 64 \\
Control 1 & 60 & Female & NA \\
Control 2 & 51 & Female & NA \\
Control 3 & 64 & Female & NA \\
Control 4 & 54 & Female & NA \\
Control 5 & 46 & Female & NA \\
Control 6 & 55 & Female & NA \\
\hline
\end{tabular}

Patients in the study group had a high preoperative PASP ( $>40 \mathrm{mmHg}$ ). PASP, pulmonary artery systolic pressure; NA, not applicable.

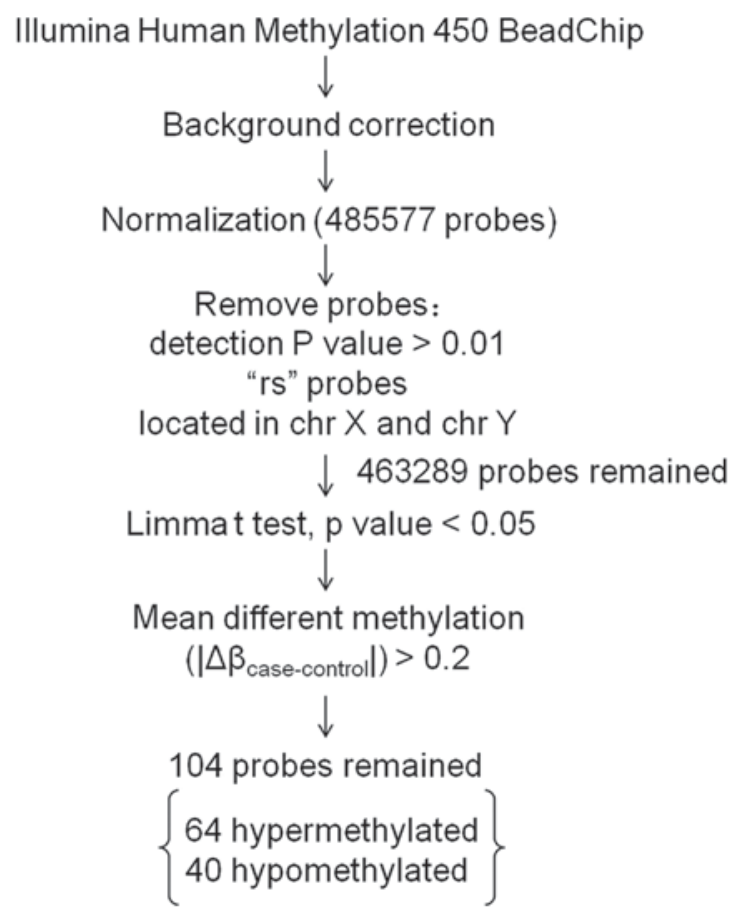

Figure 1. Workflow of the identification of differentially methylation regions by array analyses of 12 samples. rs, random single nucleotide polymorphisms; chr, chromosome.

the $450 \mathrm{k}$ chip platform, including mappings between gene symbol identifiers and manufacturer identifiers and genomic positions. All annotations are based on human genome build 19. A heatmap was created via heatmap. 2 function in the gplots package. Signaling pathway and gene ontology (GO) enrichment analyses were conducted using the Database for 

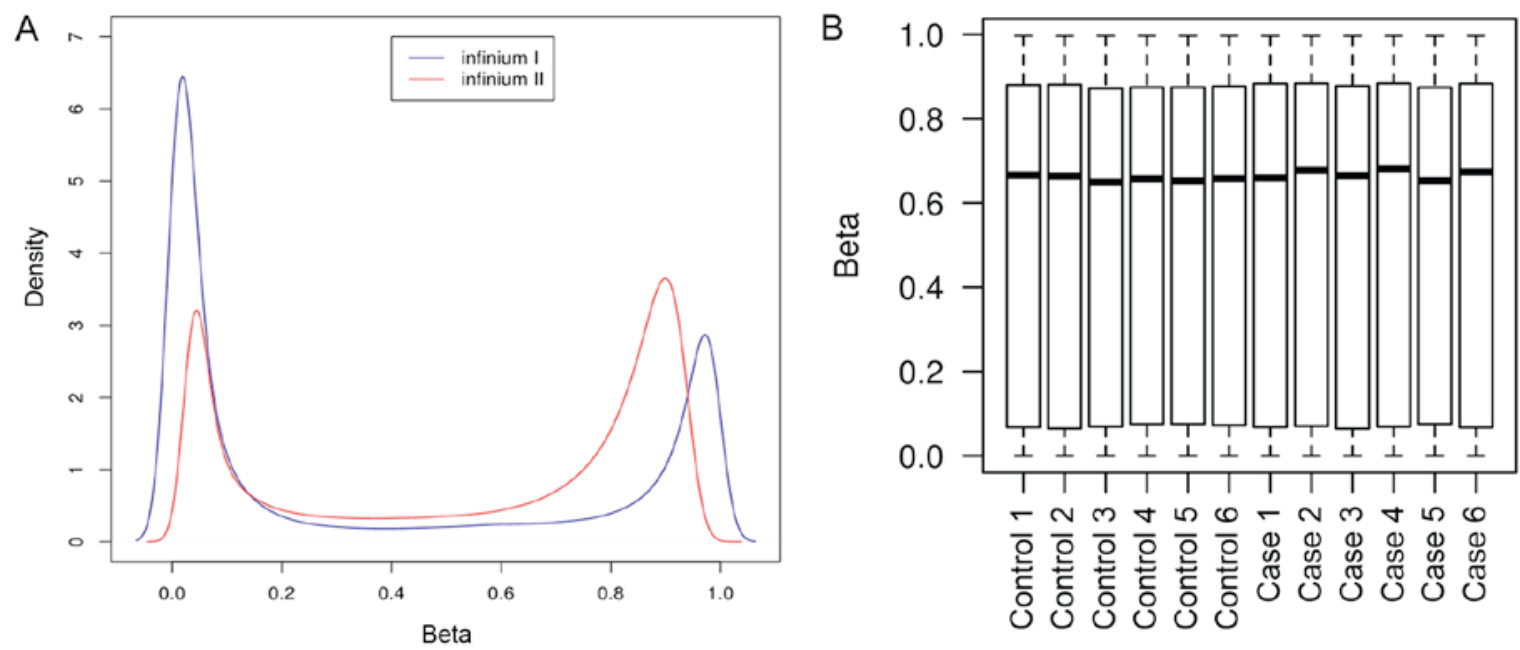

Figure 2. Quality control of methylation data. (A) Density plots of the $\beta$-values for each Infinium assay type considered (blue: Infinium I; red: Infinium II), Infinium I assay: 2 bead types per CpG locus, both in the same color channel; Infinium II assay: 1 bead type per CpG locus, two color readout. (B) Boxplots of $\beta$-values in the 6 controls and 6 patients with rheumatic heart disease and secondary pulmonary arterial hypertension.

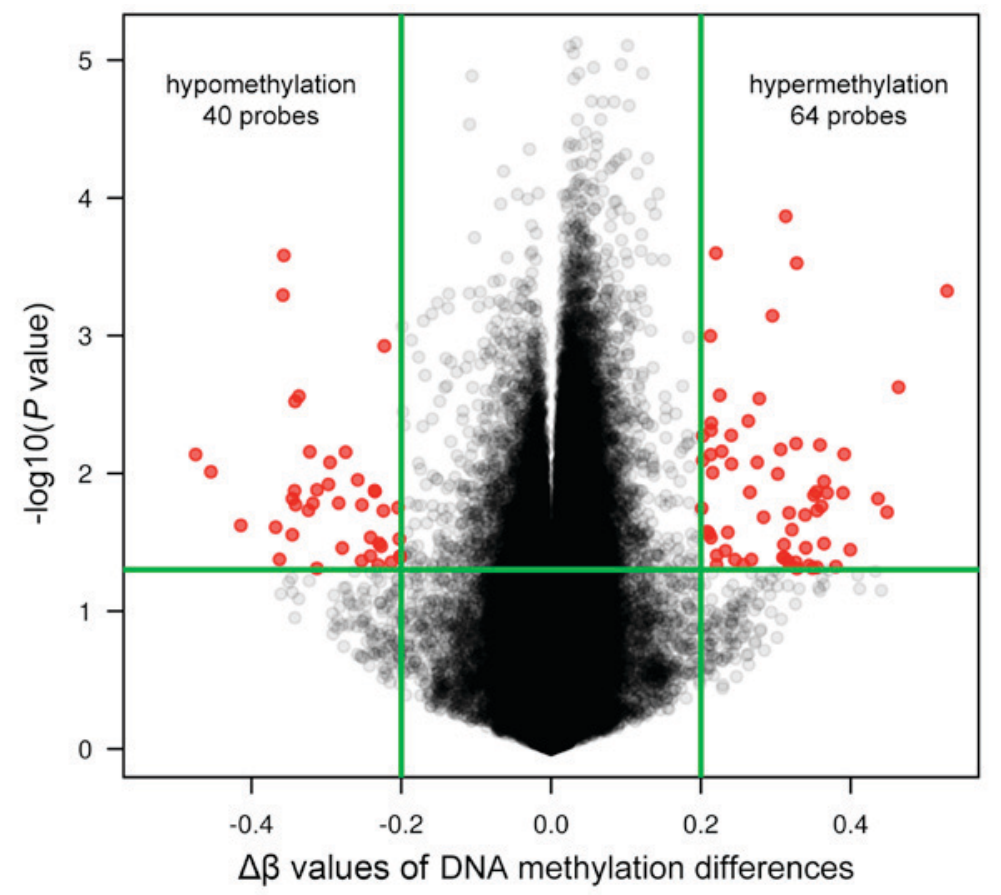

Figure 3. Volcano plot of DMRs in the study group compared with the control group. Green lines represent the restricted conditions for discovering DMRs. Red dots indicate significant results $(\mathrm{P}<0.05$ and an absolute value of $\Delta \beta>0.2)$. DMR, differentially methylated regions.

Annotation, Visualization and Integrated Discovery (DAVID; version 6.7; http://david.abcc.ncifcrf.gov) and the Kyoto Encyclopedia of Genes and Genomes (KEGG; www.genome. $\mathrm{jp} / \mathrm{kegg}$ ), respectively.

\section{Results}

Quality control of methylation data. Genome-wide DNA methylation profiles of the 12 samples were generated using the Infinium HumanMethylation450 BeadChip kit (Fig. 1). The density distribution of the $\beta$-values is presented in Fig. $2 \mathrm{~A}$. The results revealed a typical bimodal-shape distribution of $\beta$-values. Furthermore, boxplots of $\beta$-value distributions demonstrated homogenous levels of methylation across all of the samples (Fig. 2B).

DMRs between patients with RHD-PAH and healthy controls. The graph in Fig. 3 represents the distribution of $\mathrm{CpG}$ sites sorted by mean $\Delta \beta$ values and P-values (Fig. 3). In the RHD-PAH group there were 40 hypomethylated and 64 hypermethylated $\mathrm{CpG}$ sites compared with the healthy controls. These 104 identified DMRs were mapped to 60 known genes (data not shown), such as protein kinase $\mathrm{C}$ alpha (PRKCA), fibroblast growth factor receptor 2, protamine 1, S-phase kinase associated protein 2 and hyperpolarization activated cyclic nucleotide gated potassium channel 2. A total of 17 (16.35\%) of the DMRs were in promoter 


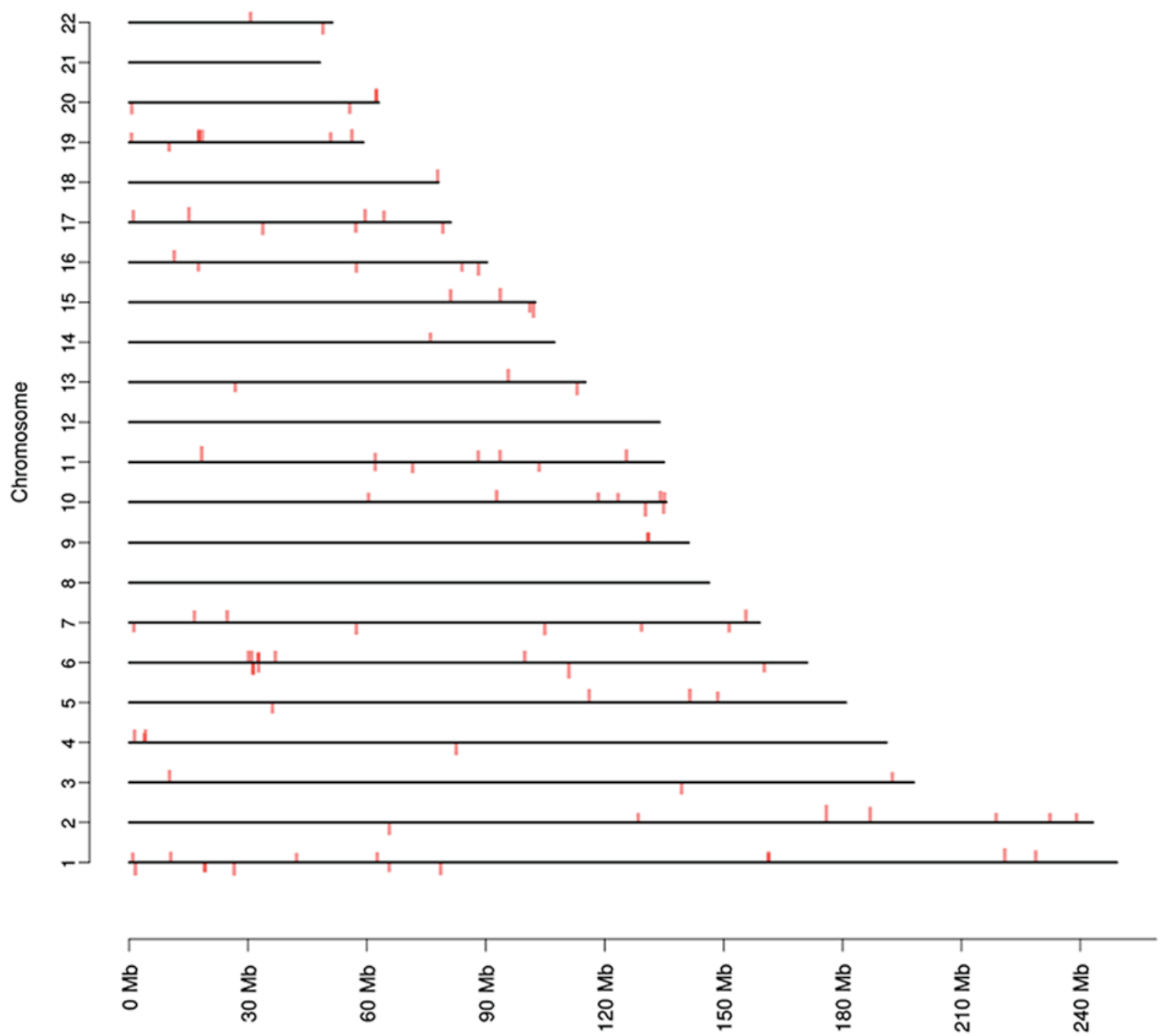

Figure 4. Chromosomal distribution of significant DMRs between the study and control groups. Red lines represent DMRs. The length of the line represents the $\Delta \beta$ value. Upward lines are hypermethylated CpG sites and downward lines are hypomethylated CpG sites. DMR, differentially methylated regions.

regions (5'UTR, TSS200, TSS1500 and 1stExon), 41 (39.42\%) in gene body regions, 5 (4.81\%) in 3'UTR regions and $41(39.42 \%)$ were unknown.

The chromosomal distribution of DMRs was evaluated according to the annotation of genomic positions. It was revealed to affect a large range of genes and chromosomal regions (Fig. 4). Patient samples were also ordered by hierarchical clustering, and a heat map was produced to allow the visualization of the hypermethylated DMRs associated with RHD-PAH (Fig. 5). The DMRs were mapped to a number of genes, including $P R K C A$, protein kinase AMP-activated non-catalytic subunit $\gamma 2$ (PRKAG2), sprouty related EVH1 domain containing 2 (SPRED2) and LIF interleukin 6 family cytokine $(L I F)$.

Enrichment analysis of the genes with DMRs. DAVID GO enrichment analysis of the genes that the DMRs were mapped to revealed several significantly enriched GO terms, including negative regulation of protein kinase activity, positive regulation of protein amino acid phosphorylation, negative regulation of transferase activity and positive regulation of phosphate metabolic process $(\mathrm{P}<0.05$; Table II). PRKCA, PRKAG2, SPRED2 and LIF were each enriched in at least two of these processes. No significantly enriched signaling pathways were identified by KEGG.

\section{Discussion}

In the present study, a genome-wide high-throughput assay was used to identify DMRs in Chinese patients with RHD-PAH. Rheumatic diseases, including RHD, frequently possess an element of autoimmunity (22). The hypomethylation of immune response-associated genes (cluster of differentiation 9, matrix metallopeptidase 9, platelet derived growth factor receptor $\alpha$ and bone marrow stromal cell antigen 2) has previously been identified in systemic lupus erythematosus (23), and DMRs in osteoarthritis are primarily associated with inflammatory/defensive immune responses (24). It has also been reported that the altered DNA methylation of genes, including interleukin (IL) 6 receptor, calpain 8, dipeptidyl 
Table II. GO term enrichment analysis of the differentially methylated regions in patients with rheumatic heart disease and secondary pulmonary arterial hypertension compared with the healthy controls.

\begin{tabular}{llll}
\hline Enriched term & \multicolumn{1}{c}{ Description } & P-value & \multicolumn{1}{c}{ Genes involved } \\
\hline GO:0006469 & Negative regulation of protein kinase activity & 0.031 & PRKCA, PRKAG2, SPRED2 \\
GO:0001934 & Positive regulation of protein amino acid phosphorylation & 0.032 & PRKCA, LIF, PRKAG2 \\
GO:0051348 & Negative regulation of transferase activity & 0.037 & PRKCA, PRKAG2, SPRED2 \\
GO:0045937 & Positive regulation of phosphate metabolic process & 0.040 & PRKCA, LIF, PRKAG2 \\
\hline
\end{tabular}

GO, gene ontology; PRKCA, protein kinase $\mathrm{C} \alpha ; P R K A G 2$, protein kinase AMP-activated non-catalytic subunit $\gamma 2$ 2; SPRED2, sprouty related EVH1 domain containing 2; LIF, LIF interleukin 6 family cytokine.

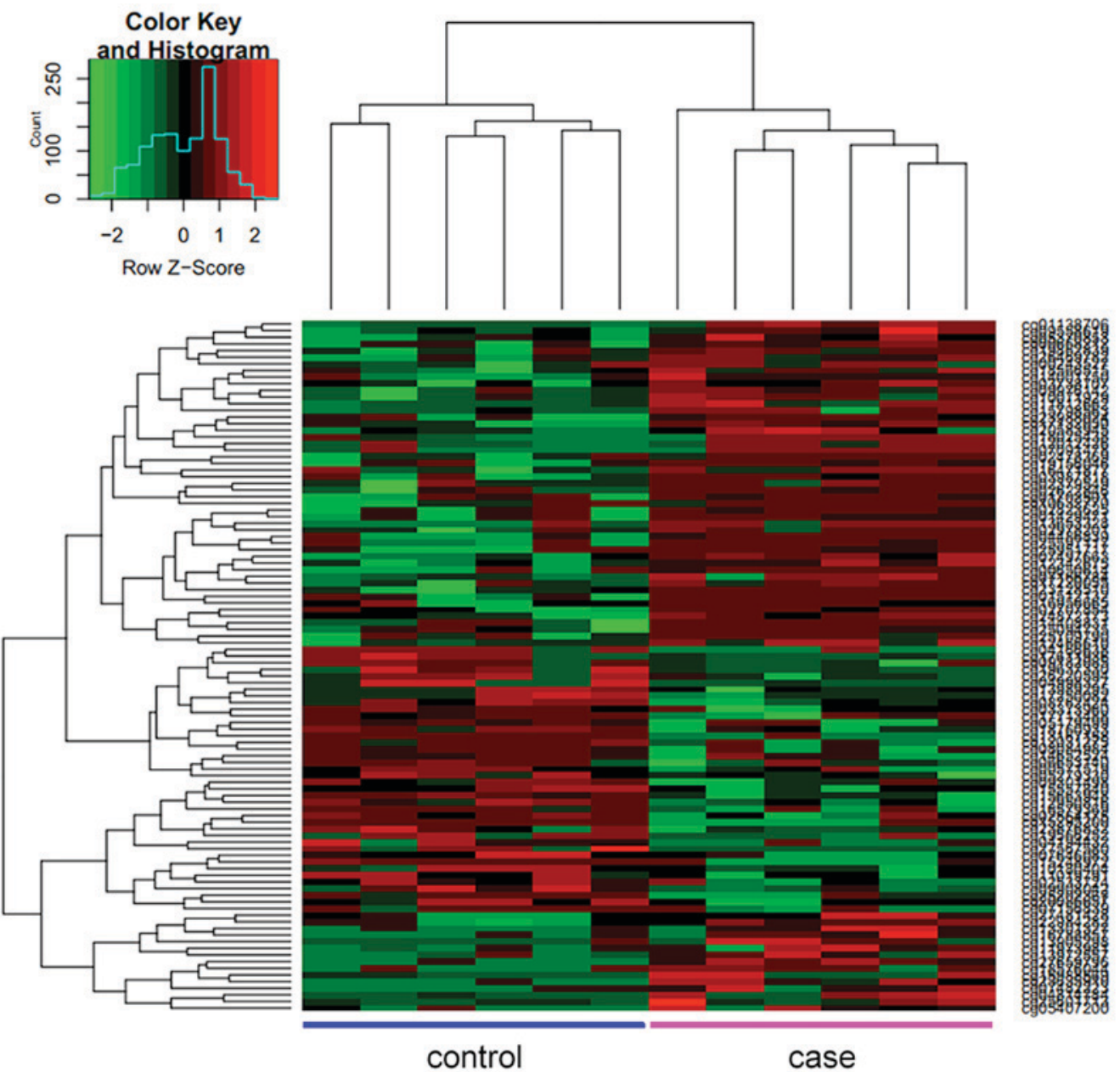

Figure 5. Heat map of differentially methylated CpG sites in the study group compared with the control group. DNA Green denotes CpGs with the lowest methylation levels and red denotes CpGs with the highest methylation levels.

peptidase 4 and multiple homeobox genes, may mediate the risk of rheumatoid arthritis (25).

In the present study, four candidate genes (PRKCA, LIF, $P R K A G 2$ and SPRED2) were identified to be associated with the pathogenesis of RHD. Differentially methylated PRKCA contributes to the risk of developing fibromyalgia in women (26). Patients with fibromyalgia typically suffer from rheumatic symptoms, which are likely to be associated with inflammatory cytokines (27). $L I F$, which encodes a member of the IL- 6 cytokine family, downregulates autoimmune responses by enhancing the number of regulatory $\mathrm{T}$ cells (28). This suggests that the silencing of $L I F$ by hypermethylation may increase the risk of RHD. PRKAG2 mutation is responsible for glycogen storage disease of the heart $(29,30)$. However, the role of PRKAG2 in the development RHD-PAH remains unclear. SPRED2 has been reported to be a repressor of immune responses (31). The SPRED2 rs934734 polymorphism was identified to be significantly associated with an increased risk of rheumatoid arthritis (32), which suggests that SPRED2 may serve a role in the pathogenesis of RHD. However, further 
studies are required to explore the underlying mechanisms of these DMRs in the pathogenesis of RHD-PAH.

The present study had several limitations. The results of the current study were based on a genome-wide methylation array of 6 patients with RHD-PAH and 6 healthy controls, and large population validation for clinical application should be performed. Furthermore, the present study only included female Chinese patients with RHD-PAH, and so the DMRs identified here require confirmation in males and patients of different ethnicities. Finally, although DNA methylation regulation is an important mechanism in the pathogenesis of RHD-PAH, the involvement of other epigenetic regulation, including histone modification and the effects of miRNA, remains to be explored.

In conclusion, the results of the present study identified 40 hypomethylated and 64 hypermethylated $\mathrm{CpG}$ sites between the RHD-PAH group and the control group. These DMRs may provide novel insights into the pathogenesis of RHD-PAH.

\section{Acknowledgements}

The present study was supported by the National Natural Science Foundation of China (grant no. 81371469), Zhejiang Provincial Natural Science Foundation (grant no. LY14H160008), Ningbo City Medical Science and Technology Projects (grant no. 2014A20), the Advanced Key Scientific and Technological Programs of Ningbo (grant no. 2012C5017), the Natural Science Foundation of Ningbo (grant no. 2014A610272), the Natural Science Foundation of Ningbo (grant no. 2016A610197), the Science and Technology Foundation of Ningbo (grant no. 2016C51012) and the Science and Technology Innovation Team of Ningbo (grant no. 2011B82015).

\section{References}

1. Villa-Forte A and Mandell BF: Cardiovascular disorders and rheumatic disease. Rev Esp Cardiol 64: 809-817, 2011 (In Spanish).

2. Marijon E, Mirabel M, Celermajer DS and Jouven X: Rheumatic heart disease. Lancet 379: 953-964, 2012.

3. Li N, Lian J, Zhao S, Zheng D, Yang X, Huang X, Shi X, Sun L, Zhou Q, Shi H, et al: Detection of differentially expressed micrornas in rheumatic heart disease: miR-1183 and miR-1299 as potential diagnostic biomarkers. Biomed Res Int 2015: 524519, 2015.

4. Lu H, Pan WZ, Wan Q, Cheng LL, Shu XH, Pan CZ, Qian JY and Ge JB: Trends in the prevalence of heart diseases over a ten-year period from single-center observations based on a large echocardiographic database. J Zhejiang Univ Sci B 17: 54-59, 2016.

5. Victor S: Dilemmas in the management of rheumatic heart disease. J Indian Med Assoc 97: 265-270, 1999.

6. Sriharibabu M, Himabindu Y and Kabir Z: Rheumatic heart disease in rural south India: A clinico-observational study. J Cardiovasc Dis Res 4: 25-29, 2013.

7. Izikki M, Guignabert C, Fadel E, Humbert M, Tu L, Zadigue P, Dartevelle P, Simonneau G, Adnot S, Maitre B, et al Endothelial-derived FGF2 contributes to the progression of pulmonary hypertension in humans and rodents. J Clin Invest 119: 512-523, 2009

8. Zhao Y, Peng J, Lu C, Hsin M, Mura M, Wu L, Chu L, Zamel R, Machuca T, Waddell T, et al: Metabolomic heterogeneity of pulmonary arterial hypertension. PLoS One 9: e88727, 2014.

9. Xiaoying C, Huadan Y, Qingxiao H, Annan Z, Linlin T and Shiwei D: The effects of DNA methylation on the homeostasis in vascular diseases. Yi Chuan 37: 221-232, 2015 (In Chinese).

10. Soubrier F, Chung WK, Machado R, Grünig E, Aldred M, Geraci M, Loyd JE, Elliott CG, Trembath RC, Newman JH and Humbert M: Genetics and genomics of pulmonary arterial hypertension. J Am Coll Cardiol 62 (25 Suppl): D13-D21, 2013.
11. Kim JD, Lee A, Choi J, Park Y, Kang H, Chang W, Lee MS and Kim J: Epigenetic modulation as a therapeutic approach for pulmonary arterial hypertension. Exp Mol Med 47: e175, 2015.

12. Pousada G, Baloira A and Valverde D: Methylation analysis of the BMPR2 gene promoter region in patients with pulmonary arterial hypertension. Arch Bronconeumol 52: 293-298, 2016 (In English, Spanish).

13. Saco TV, Parthasarathy PT, Cho Y, Lockey RF and Kolliputi N: Role of epigenetics in pulmonary hypertension. Am J Physiol Cell Physiol 306: C1101-C1105, 2014.

14. Liu R, Leslie KL and Martin KA: Epigenetic regulation of smooth muscle cell plasticity. Biochim Biophys Acta 1849: 448-453, 2015.

15. Ospelt C: Epigenetic biomarkers in rheumatology-the future? Swiss Med Wkly 146: w14312, 2016.

16. Plant D, Webster A, Nair N, Oliver J, Smith SL, Eyre S, Hyrich KL, Wilson AG, Morgan AW, Isaacs JD, et al: Differential methylation as a biomarker of response to etanercept in patients with rheumatoid arthritis. Arthritis Rheumatol 68: 1353-1360, 2016.

17. Zufferey F, Williams FM and Spector TD: Epigenetics and methylation in the rheumatic diseases. Semin Arthritis Rheum 43: 692-700, 2014.

18. Xu L, Zheng D, Wang L, Jiang D, Liu H, Xu L, Liao Q, Zhang L, Liu P, Shi X, et al: GCK gene-body hypomethylation is associated with the risk of coronary heart disease. Biomed Res Int 2014: 151723,2014

19. Bibikova M, Le J, Barnes B, Saedinia-Melnyk S, Zhou L, Shen R and Gunderson KL: Genome-wide DNA methylation profiling using Infinium ${ }^{\circledR}$ assay. Epigenomics 1: 177-200, 2009.

20. Bibikova M, Barnes B, Tsan C, Ho V, Klotzle B, Le JM, Delano D, Zhang L, Schroth GP, Gunderson KL, et al: High density DNA methylation array with single $\mathrm{CpG}$ site resolution. Genomics 98: 288-295, 2011.

21. Du P, Zhang X, Huang CC, Jafari N, Kibbe WA, Hou L and Lin SM: Comparison of Beta-value and M-value methods for quantifying methylation levels by microarray analysis. BMC Bioinformatics 11: 587, 2010.

22. Bright PD, Mayosi BM and Martin WJ: An immunological perspective on rheumatic heart disease pathogenesis: More questions than answers. Heart 102: 1527-1532, 2016.

23. Jeffries MA, Dozmorov M, Tang Y, Merrill JT, Wren JD and Sawalha AH: Genome-wide DNA methylation patterns in CD4+ T cells from patients with systemic lupus erythematosus. Epigenetics 6: 593-601, 2011.

24. Fernández-Tajes J, Soto-Hermida A, Vázquez-Mosquera ME, Cortés-Pereira E, Mosquera A, Fernández-Moreno M, Oreiro N, Fernández-López C, Fernández JL, Rego-Pérez I and Blanco FJ: Genome-wide DNA methylation analysis of articular chondrocytes reveals a cluster of osteoarthritic patients. Ann Rheum Dis 73: 668-677, 2014

25. de la Rica L, Urquiza JM, Gómez-Cabrero D, Islam AB, López-Bigas N, Tegnér J, Toes RE and Ballestar E: Identification of novel markers in rheumatoid arthritis through integrated analysis of DNA methylation and microRNA expression. J Autoimmun 41: 6-16, 2013.

26. Menzies V, Lyon DE, Archer KJ, Zhou Q, Brumelle J, Jones KH, Gao G, York TP and Jackson-Cook C: Epigenetic alterations and an increased frequency of micronuclei in women with fibromyalgia. Nurs Res Pract 2013: 795784, 2013.

27. Garcia JJ and Ortega E: Soluble fractalkine in the plasma of fibromyalgia patients. An Acad Bras Cienc 86: 1915-1917, 2014.

28. Janssens K, Van den Haute C, Baekelandt V, Lucas S, van Horssen J, Somers V, Van Wijmeersch B, Stinissen P, Hendriks JJ, Slaets $\mathrm{H}$ and Hellings $\mathrm{N}$ : Leukemia inhibitory factor tips the immune balance towards regulatory $\mathrm{T}$ cells in multiple sclerosis. Brain Behav Immun 45: 180-188, 2015.

29. Thevenon J, Laurent G, Ader F, Laforêt P, Klug D, Duva Pentiah A, Gouya L, Maurage CA, Kacet S, Eicher JC, et al: High prevalence of arrhythmic and myocardial complications in patients with cardiac glycogenosis due to PRKAG2 mutations. Europace: pii: euw067, 2016 (Epub ahead of print).

30. Hedberg-Oldfors $\mathrm{C}$ and Oldfors A: Polyglucosan storage myopathies. Mol Aspects Med 46: 85-100, 2015.

31. Kidane YH, Lawrence C and Murali TM: Computational approaches for discovery of common immunomodulators in fungal infections: Towards broad-spectrum immunotherapeutic interventions. BMC Microbiol 13: 224, 2013.

32. Stahl EA, Raychaudhuri S, Remmers EF, Xie G, Eyre S, Thomson BP,Li Y, Kurreeman FA, Zhernakova A, Hinks A, et al: Genome-wide association study meta-analysis identifies seven new rheumatoid arthritis risk loci. Nat Genet 42: 508-514, 2010. 